\title{
Non variational elliptic systems in dimension two: a priori bounds and existence of positive solutions
}

\author{
Djairo G. de Figueiredo \\ IMECC - Departamento de Matemática, \\ Universidade Estadual de Campinas, 13083-970 Campinas, S.P., Brazil \\ e-mail: djairo@ime.unicamp.br \\ João Marcos do Ó \\ CCEN - Departamento de Matemática, \\ Universidade Federal da Paraíba, 58059.900 João Pessoa, Pb, Brazil \\ e-mail: jmbo@mat.ufpb.br \\ Bernhard Ruf \\ Dipartimento di Matematica, \\ Università degli Studi, Via Saldini 50, 20133 Milano, Italy \\ e-mail: ruf@mat.unimi.it
}

Dedicated to Felix Browder on the occasion of his 80th birthday

\section{Abstract}

We establish a priori bounds for positive solutions of semilinear elliptic systems of the form

$$
\left\{\begin{array}{c}
-\Delta u=g(x, u, v), \text { in } \Omega \\
-\Delta v=f(x, u, v), \text { in } \Omega \\
u>0, v>0 \text { in } \Omega \\
u=v=0 \text { on } \partial \Omega
\end{array}\right.
$$

where $\Omega$ is a bounded and smooth domain in $\mathbb{R}^{2}$. We obtain results concerning such bounds when $f$ and $g$ depend exponentially with respect to $u$ and $v$. Based on these bounds, existence of positive solutions is proved. 
1991 Mathematics Subject Classification. 35J60, 35B45.

Key words. Elliptic systems, A priori estimates. 


\section{Introduction}

In this paper we establish the existence of positive solutions for semilinear elliptic systems of the form

$$
\left\{\begin{array}{c}
-\Delta u=g(x, u, v), \text { in } \Omega \\
-\Delta v=f(x, u, v), \text { in } \Omega \\
u>0, v>0 \text { in } \Omega \\
u=v=0 \text { on } \partial \Omega
\end{array}\right.
$$

where $\Omega$ is a bounded domain in $\mathbb{R}^{2}$ with smooth boundary $\partial \Omega, \Delta$ is the Laplace operator, and $f, g: \Omega \times \mathbb{R}^{2} \rightarrow \mathbb{R}$ are continuous functions.

This class of systems has been extensively studied when $\Omega$ is a bounded domain in $\mathbb{R}^{N}$, with $N \geq 3$, see the recent survey paper [7].

The case of dimension 2 presents some different features than the case of higher dimensions, and has been less studied. Some material in the case of dimension two has been collected in the recent survey paper [18]. Using variational methods, systems of the form

$$
\left\{\begin{array}{c}
-\Delta u=g(x, v), \text { in } \Omega \\
-\Delta v=f(x, u), \text { in } \Omega \\
u>0, v>0 \text { in } \Omega \\
u=v=0 \text { on } \partial \Omega
\end{array}\right.
$$

have been treated in [11]. Existence of solutions to (1.2) were obtained for nonlinearities both sub-critical and critical in the sense of the TrudingerMoser exponential growth.

Later, in [13], we investigated the question of a priori bounds for positive solutions of (1.2). Such bounds were obtained under much more restrictive hypotheses on the growth of the nonlinearities as compared with the above growths used in the variational approach.

This fact is in agreement with a similar phenomenon already observed in the scalar case by the authors of [5] and [6]. We recall that existence of solutions for the scalar problem

$$
\left\{\begin{array}{c}
-\Delta u=f(x, u), \text { in } \Omega \\
u>0 \text { in } \Omega \\
u=0 \text { on } \partial \Omega
\end{array}\right.
$$


can be proved for cases where the non-linearity $f(x, u)$ behaves at $\infty$ as $e^{\alpha|u|^{2}}$ (plus some additional conditions needed to use variational methods). However, the a priori bounds for positive solutions of (1.3) have been obtained in [5] and [6] under more restrictive conditions, namely $f$ behaving as $e^{u}$ at $\infty$. And in [5] it is even shown that in general one cannot expect to get the bounds if $f$ has higher growths in the non-homogeneous case.

It is apparent that one is in the presence of similar phenomena observed in [19] in dimension $N \geq 3$. They have shown that a priori bounds in these dimensions, for general non-homogeneous nonlinearities, can be obtained only if $f(x, u)$ has polynomial growth at $\infty$ like $u^{p}$ with $p \leq \frac{N+1}{N-1}$; this is the so-called Brezis-Turner exponent, which is the best exponent to get a priori bounds for positive solutions of (1.3) using Hardy inequality, see [3].

The purpose of the present paper is to study systems of form (1.1) which are not necessarily variational. We will derive a priori estimates for positive solutions for nonlinearities whose growths will be restricted by exponentials whose exponents are related and compensate each other. Then we use topological methods to prove the existence of positive solutions for these systems.

We assume throughout the paper that the nonlinearities satisfy the hypothesis:

$\left(H_{0}\right) \quad f, g: \Omega \times \mathbb{R} \times \mathbb{R} \rightarrow \mathbb{R}^{+}$are continuous.

To start with, we consider very weak solutions of system (1.1), that is solutions in the sense of distributions. More precisely, we assume that $u$ and $v$ are $L^{1}$-functions such that

$$
f(x, u, v) \text { and } g(x, u, v) \in L^{1}(\Omega)
$$

and

$$
\begin{aligned}
& -\int_{\Omega} u \Delta \varphi \mathrm{d} x=\int_{\Omega} g(x, u, v) \varphi \mathrm{d} x \\
& -\int_{\Omega} v \Delta \varphi \mathrm{d} x=\int_{\Omega} f(x, u, v) \varphi \mathrm{d} x
\end{aligned} \quad, \quad \forall \varphi \in E=\left\{u \in C^{2}(\bar{\Omega}),\left.u\right|_{\partial \Omega}=0\right\} .
$$

Before stating our main results on a priori bounds, we present a result on the regularity of the distribution solutions of (1.1). For that matter, a growth assumption on only one of the nonlinearities suffices, namely,

$$
\left(H_{1}\right) \quad f(x, t, s) \leq c e^{p t+q s} \text { and } g(x, t, s) \leq e^{p^{t} t} h(s)
$$


or

$$
\left(H_{1}^{\prime}\right) \quad g(x, t, s) \leq c e^{p t+q s} \text { and } f(x, t, s) \leq e^{q^{\prime} s} k(t)
$$

where $c, p, q, p^{\prime}$ and $q^{\prime}$ are some positive constants, and $h(s)$ and $k(t)$ are positive, continuous functions.

Theorem 1.1 (Regularity of distribution solutions) Assume $\left(H_{1}\right)$ or $\left(H_{1}^{\prime}\right)$. Then the distribution solutions of system (1.1) are in fact in $L^{\infty}(\Omega)$.

It follows from Theorem 1.1 that any solution $(u, v)$ of system (1.1) satisfies $\int_{\Omega} g(x, u, v) \mathrm{d} x<\infty, \int_{\Omega} f(x, u, v) \mathrm{d} x<\infty$. Our next result states that under some additional hypotheses there is a uniform bound for these integrals.

Let $c(x) \in C^{(\bar{\Omega})}$ be a positive function and denote by $\lambda_{1}(c)$ the first eigenvalue of the following eigenvalue problem

$$
-\Delta \varphi=\lambda c(x) \varphi, \text { in } \Omega, \varphi=0 \text {, on } \partial \Omega \text {. }
$$

We now state the "superlinearity" assumptions we need.

$\left(H_{2}\right)$ There exist positive functions $a, b \in C(\bar{\Omega})$ and constants $a_{1}, b_{1}$ with the property:

$$
f(x, t, s) \geq a(x) t-a_{1} \text { and } g(x, t, s) \geq b(x) s-b_{1}
$$

and such that

$$
a(x) \lambda_{1}(a)^{2}<b(x), x \in \bar{\Omega} .
$$

$\left(H_{2}^{\prime}\right)$ There exist positive functions $a, b \in C(\bar{\Omega})$ and constants $a_{1}, b_{1}$ with the property

$$
f(x, t, s) \geq a(x) t-a_{1} \text { and } g(x, t, s) \geq b(x) s-b_{1}
$$

and such that

$$
b(x) \lambda_{1}(b)^{2}<a(x), x \in \bar{\Omega} .
$$

The next assumption concerns the behaviour of the nonlinearities near the boundary:

$\left(H_{3}\right)$ For some $r>0$,

$$
\frac{\partial}{\partial t} f(x, t, s) \geq 0 \text { and } \frac{\partial}{\partial s} g(x, t, s) \geq 0 \text { in } \Omega_{r} \times \mathbb{R} \times \mathbb{R},
$$

where $\Omega_{r}:=\{x \in \bar{\Omega}: \operatorname{dist}(x, \partial \bar{\Omega}) \leq r\}$. 
We note that condition $\left(\mathrm{H}_{3}\right)$ will allow the use of the Maximum Principle for cooperative systems; this is a basic tool in order to apply the Moving Planes technique (cf. [9], [20]).

Due to the fact that we are considering non-autonomous problems, we also need geometric assumptions concerning the behavior of $f$ and $g$ with respect to $x$ near the boundary.

$\left(H_{4}\right)$ ( $\Omega$ a convex domain) There exist $r, \delta>0$ such that $g(\cdot, t, s), f(\cdot, t, s) \in$ $C^{1}\left(\Omega_{r}\right)$, and

$$
\nabla_{x} g(x, t, s) \cdot \theta \leq 0 \text { and } \nabla_{x} f(x, t, s) \cdot \theta \leq 0
$$

for all $x \in \Omega_{r}, t, s \geq 0$, and unit vectors $\theta$ such that $|\theta-\nu(\bar{x})|<\delta$, where $\bar{x}$ is the closest point to $x$ in $\partial \Omega$ and $\nu(\bar{x})$ denotes the unit external normal to $\partial \Omega$ in the point $\bar{x}$.

If $\Omega$ is not convex, we use the Kelvin transform as in [6] to reduce the problem to a situation as in the convex case. So we follow [6] and assume

$\left(H_{5}\right)$ ( $\Omega$ a general domain) There exist $r, C>0$ such that $g(\cdot, t, s)$, $f(\cdot, t, s) \in C^{1}\left(\Omega_{r}\right)$ and

$$
\left|\nabla_{x} g(x, t, s)\right| \leq C g(x, t, s) \text { and }\left|\nabla_{x} f(x, t)\right| \leq C f(x, t, s) .
$$

for all $x \in \Omega_{r}$ and $t, s \geq 0$.

Theorem 1.2 (Uniform Estimates) Assume $\left(H_{1}\right)$ (or $\left.\left(H_{1}^{\prime}\right)\right),\left(H_{2}\right)$ (or $\left.\left(H_{2}^{\prime}\right)\right),\left(H_{3}\right),\left(H_{4}\right)$ and $\Omega$ convex (or $\left.\left(H_{5}\right)\right)$. Then there exists a positive constant $C$, depending only on $f, g$ and $\Omega$, such that

$$
\int_{\Omega} g(x, u, v) \mathrm{d} x \leq C, \int_{\Omega} f(x, u, v) \mathrm{d} x \leq C,
$$

for all $(u, v)$ solution of system (1.1).

In order to obtain a priori bounds for the solutions of system (1.1) we have to assume further conditions regarding the growth at infinity of the nonlinearities $f$ and $g$. For that matter, we introduce the following conditions. 
$\left(H_{6}\right)$ There exists a constant $c$ such that

$$
g(x, t, s) \leq c e^{t^{\alpha}+s^{\beta}} \quad \text { and } \quad f(x, t, s) \leq c e^{t^{\beta^{\prime}}+s^{\alpha^{\prime}}},
$$

where

$$
\begin{array}{ll}
\text { a) } & 0 \leq \alpha \leq \beta \text { and } 0 \leq \alpha^{\prime} \leq \beta^{\prime} \\
\text { b) } & \alpha+\beta<2 \text { and } \alpha^{\prime}+\beta^{\prime}<2 \\
\text { c) } & \beta+\beta^{\prime}<2
\end{array}
$$

Remark 1.1 From our main growth assumption $\left(H_{6}\right)$ it follows that both $\left(H_{1}\right)$ and $\left(H_{1}^{\prime}\right)$ are satisfied.

Example 1.1 The nonlinearities below satisfy condition $\left(H_{6}\right)$ :

$$
f(x, t, s)=e^{t+s^{1 / 2}}, g(x, t, s)=e^{t^{1 / 2}+s^{3 / 4}}
$$

Remark 1.2 If $f(x, t, s) \leq c^{\prime} e^{p t^{\alpha}+q t^{\beta}}$, for some $p, q>1$, then condition $\left(H_{6}\right)$ will be satisfied for suitable choices of $c$ and $\alpha<\tilde{\alpha}<1, \beta<\tilde{\beta}<1$, and similarly for $g(x, t, s)$. So these conditions are not more general than $\left(H_{6}\right)$.

The next Theorem gives a priori estimates for positive solutions of system (1.1) under the growth restrictions $\left(H_{6}\right)$ :

Theorem 1.3 Assume $\left(H_{2}\right)$ (or $\left.\left(H_{2}^{\prime}\right)\right),\left(H_{3}\right),\left(H_{4}\right)$ and $\Omega$ convex $\left(\right.$ or $\left.\left(H_{5}\right)\right)$, and $\left(H_{6}\right)$. Then there exists a constant $C>0$ such that

$$
\|u\|_{L^{\infty}} \leq C \text { and }\|v\|_{L^{\infty}} \leq C
$$

for all solutions $(u, v)$ of system (1.1).

\section{Remark 1.3}

1) Condition $\left(H_{6}, a\right)$ says that the system is strongly coupled.

2) If $\alpha=0=\alpha^{\prime}$, then the system reduces to

$$
-\Delta u=g(x, v) \quad, \quad-\Delta v=f(x, u)
$$

which was considered in [12]; indeed, in this case Theorem 1.3 contains and generalizes Theorems 1.4 and 1.5 from that article, which covers the case $\beta+\beta^{\prime}<2$. 
3) The general case $\beta+\beta^{\prime}=2$ (and $\alpha=\alpha^{\prime}=0$ ) was left open in [12], except for the particular case $\beta=\beta^{\prime}=1$, for which it was possible to obtain a priori bounds following the techniques developed by Brezis-Merle [5].

Here we come back to this situation, which we call "critical growth", and generalize it to the case where both nonlinearities depend on the variables $u$ and $v$, both with exponential growth, i.e. with $\beta=\beta^{\prime}=1$ together with $\alpha=\alpha^{\prime}=1$; see the next result.

Theorem 1.4 Assume $\left(H_{2}\right)$ (or $\left.\left(H_{2}^{\prime}\right)\right),\left(H_{3}\right),\left(H_{4}\right)$ and $\Omega$ convex $\left(\right.$ or $\left.\left(H_{5}\right)\right)$, and assume that $f(x, t, s)$ and $g(x, t, s)$ satisfy:

$\left(H_{7}\right)$ There exist constants $c, d>0$ such that

$$
c e^{t+s} \leq g(x, t, s) \leq d e^{t+s}
$$

and

$$
0 \leq f(x, t, s) \leq d e^{t+s} .
$$

Then all positive solutions $(u, v)$ of system (1.1) are uniformly bounded in $L^{\infty}$.

\section{Example 1.2}

$$
g(x, t, s)=b e^{t+s} \quad, \quad f(x, t, s)=\sin ^{2}(x) e^{t+s},
$$

where $b>\sin ^{2}(x) \cdot \lambda_{1}^{2}\left(\sin ^{2}(x)\right)$, see $\left(H_{2}\right)$.

Finally, we give an existence theorem. Denote with

$$
\begin{aligned}
(-\Delta)^{-1}: C^{0}(\bar{\Omega}) & \rightarrow C^{0}(\bar{\Omega}) \\
y & \mapsto u \quad \text { s.th. }-\Delta u=y,\left.u\right|_{\partial \Omega}=0
\end{aligned}
$$

the inverse Laplace operator, with $M$ such that

$$
\left\|(-\Delta)^{-1} y\right\|_{C^{0}} \leq M\|y\|_{C^{0}}, \forall y \in C^{0}(\bar{\Omega}) .
$$

Theorem 1.5 Assume that $f$ and $g$ satisfy the hypotheses of Theorem 1.3 or Theorem 1.4, and in addition:

$\left(H_{8}\right)$ There exists $\varepsilon>0$ and constants $c_{1}, c_{2}$ with the property:

$$
\begin{aligned}
& f(x, t, s) \leq c_{1}(|t|+|s|) \text { and } g(x, t, s) \leq c_{2}(|t|+|s|), \text { for }|t|+|s| \leq \varepsilon, \\
& \text { with } M\left(c_{1}+c_{2}\right)<1 .
\end{aligned}
$$

Then system (1.1) has a nontrivial solution $(u, v)$. 


\section{Regularity of distribution solutions}

For easy reference, we state a result due to Brezis-Merle [5] which will be used to prove Theorem 1.1 above, and also in the proofs of Theorems 1.3 and 1.4 .

Proposition 2.1 (Brezis-Merle) Let $u$ be a distribution solution of the linear equation

$$
\left\{\begin{aligned}
-\Delta u & =h(x) & & \text { in } \Omega \\
u & =0 & & \text { on } \partial \Omega,
\end{aligned}\right.
$$

where $\Omega$ is a bounded domain in $\mathbb{R}^{2}$, and $h \in L^{1}(\Omega)$. Then

i) for every $\delta \in(0,4 \pi)$, we have

$$
\int_{\Omega} \exp \left(\frac{(4 \pi-\delta)|u(x)|}{\|h\|_{L^{1}}}\right) \mathrm{d} x \leq \frac{4 \pi^{2}}{\delta}(\operatorname{diam} \Omega)^{2} .
$$

ii) for every $k>0, e^{k u} \in L^{1}(\Omega)$.

Proof of Theorem 1.1. Let $(u, v)$ be a distributional solution of system (1.1). By Proposition 2.1, ii) follows that $e^{u}, e^{v}$ are in $L^{p}$ for every $p \geq 1$. Consequently, if we assume $\left(H_{1}\right)$, i.e. $f(x, u, v) \leq c e^{p u+q v}$, then

$\int_{\Omega}|f(x, u, v)|^{\alpha} \mathrm{d} x \leq c \int_{\Omega} e^{\alpha p u} \cdot e^{\alpha q v} \mathrm{~d} x \leq\left(\int_{\Omega} e^{2 \alpha p u} \mathrm{~d} x\right)^{1 / 2}\left(\int_{\Omega} e^{2 \alpha q v} \mathrm{~d} x\right)^{1 / 2}$,

that is, $f(x, u, v) \in L^{\alpha}$, for every $\alpha>1$. By $L^{p}$-estimates it follows from the second equation in system (1.1) that $v \in W^{2, \alpha}(\Omega)$, for all $\alpha>1$, and hence $v \in L^{\infty}$. Using now the first equation in (1.1) we conclude that also $u \in L^{\infty}$.

Using similar arguments we come to the same conclusions, if $\left(H_{1}^{\prime}\right)$ is assumed instead of $\left(H_{1}\right)$.

Remark 2.1 As a consequence of Theorem 1.1 and standard regularity results for elliptic equations we have that solutions of (1.1) in the distribution sense are, in fact, classical solutions. 


\section{Proof of Theorem 1.2 (uniform estimates)}

Let $\varphi_{1}^{b}$ be an eigenfunction associated to the first eigenvalue $\lambda_{1}(b)$ of the eigenvalue problem

$$
-\Delta \varphi_{1}^{b}=\lambda_{1}(b) b(x) \varphi_{1}^{b} \quad \text { in } \quad \Omega \quad \text { and } \quad \varphi_{1}^{b}=0 \quad \text { on } \quad \partial \Omega
$$

which is chosen in such a way that $\varphi_{1}^{b}>0$ and $\int_{\Omega}\left|\varphi_{1}^{b}\right|^{2}=1$.

Lemma 3.1 Assume $\left(H_{1}\right)$ and $\left(H_{2}^{\prime}\right)$ (or $\left(H_{2}\right)$ ). Then we have for any solution $(u, v)$ of system (1.1)

$$
\int_{\Omega} g(x, u, v) \varphi_{1}^{b} \mathrm{~d} x \leq C, \int_{\Omega} f(x, u, v) \varphi_{1}^{b} \mathrm{~d} x \leq C,
$$

where the constant $C$ depends only on $f, g$ and $\Omega$.

Proof. From assumption $\left(H_{2}^{\prime}\right)$, it follows that one can find an $\varepsilon>0$, such that

$$
a(x) \geq\left(\lambda_{1}(b)^{2}+\varepsilon\right) b(x), \quad x \in \bar{\Omega} .
$$

Multiplying the first equation in (1.1) by $\varphi_{1}^{b}$, integrating by parts and using $\left(H_{2}^{\prime}\right)$ one obtains

$$
\begin{aligned}
\lambda_{1}(b) \int_{\Omega} u b(x) \varphi_{1}^{b} \mathrm{~d} x & =\int_{\Omega} g(x, u, v) \varphi_{1}^{b} \mathrm{~d} x \\
& \geq \int_{\Omega} b(x) v \varphi_{1}^{b} \mathrm{~d} x-\int_{\Omega} b_{1}(x) \varphi_{1}^{b} \mathrm{~d} x .
\end{aligned}
$$

Multiplying the second equation in (1.1) by $\varphi_{1}^{b}$, integrating by parts and using again $\left(H_{2}^{\prime}\right)$ one obtains

$$
\begin{aligned}
\lambda_{1}(b) \int_{\Omega} v b(x) \varphi_{1}^{b} \mathrm{~d} x & =\int_{\Omega} f(x, u, v) \varphi_{1}^{b} \mathrm{~d} x \\
& \geq \int_{\Omega} a(x) u \varphi_{1}^{b} \mathrm{~d} x-\int_{\Omega} a_{1}(x) \varphi_{1}^{b} \mathrm{~d} x .
\end{aligned}
$$

Using (3.4)and (3.2), we get

$$
\lambda_{1}(b) \int_{\Omega} v b(x) \varphi_{1}^{b} \mathrm{~d} x \geq\left(\lambda_{1}(b)^{2}+\varepsilon\right) \int_{\Omega} b(x) u \varphi_{1}^{b} \mathrm{~d} x-\int_{\Omega} a_{1}(x) \varphi_{1}^{b} \mathrm{~d} x .
$$

Next using (3.5) and (3.3) we obtain

$$
\lambda_{1}(b) \int_{\Omega} v b(x) \varphi_{1}^{b} \mathrm{~d} x \geq \frac{\left(\lambda_{1}(b)^{2}+\varepsilon\right)}{\lambda_{1}(b)} \int_{\Omega} v b(x) \varphi_{1}^{b} \mathrm{~d} x-C
$$


So it follows that $\int_{\Omega} v b(x) \varphi_{1}^{b} \leq C$, and hence by (3.4) $\int_{\Omega} f(x, u, v) \varphi_{1}^{b} \leq c$, and also $\int_{\Omega} a(x) u \varphi_{1}^{b} \leq c$ from which by (3.3) also $\int_{\Omega} g(x, u, v) \varphi_{1}^{b} \mathrm{~d} x \leq c$.

Lemma 3.2 Assume conditions $\left(H_{1}\right)$ (or $\left.\left(H_{1}^{\prime}\right)\right),\left(H_{3}\right),\left(H_{4}\right)$ and $\Omega$ convex $\left(\right.$ or $\left.\left(H_{5}\right)\right)$. Then there exist $r, \delta>0$ such that

$$
\nabla u(x) \cdot \theta \leq 0 \text { and } \nabla v(x) \cdot \theta \leq 0 \text { for all } x \in \Omega_{r},|\theta-\nu(x)|<\delta,
$$

for each $(u, v)$ solution of (1.1), where $\Omega_{r}$ is defined in $\left(H_{3}\right) ; \theta$ and $\nu$ are as in $\left(H_{4}\right)$.

Proof. We can assume, without loss of generality, that

$$
\Omega \subset \mathbb{R}_{+}^{2}:=\left\{(x, y) \in \mathbb{R}^{2}: x>0\right\} \quad \text { and } \quad(0,0) \in \partial \Omega .
$$

Now, we consider

$$
\begin{array}{lll}
T_{\lambda}:=\quad\{(x, y): x=\lambda\} & \text { the hyperplane } \\
\Sigma_{\lambda}:=\quad\{(x, y) \in \Omega: x<\lambda\} & \text { the cap } \\
\Sigma_{\lambda}^{\prime}:=\left\{\left(x_{\lambda}, y\right):(x, y) \in \Sigma_{\lambda}\right\} & \text { the reflected cap }
\end{array}
$$

where

$$
x_{\lambda}:=2 \lambda-x .
$$

It follows that there exists $\bar{\lambda}$ such that $\Sigma_{\lambda} \cup \Sigma_{\lambda}^{\prime} \subset \Omega_{r}$ for each $0<\lambda<\bar{\lambda}$. In fact this $\bar{\lambda}$ depends only on $r$ and not on the particular point on the boundary.

For $0<\lambda<\bar{\lambda}$, define in $\Sigma_{\lambda}$ the auxiliary functions

$$
\begin{aligned}
w_{\lambda}(x, y) & =u\left(x_{\lambda}, y\right)-u(x, y) \\
z_{\lambda}(x, y) & =v\left(x_{\lambda}, y\right)-v(x, y)
\end{aligned}
$$

Then we have

$$
\begin{aligned}
\Delta w_{\lambda}= & \Delta u\left(x_{\lambda}, y\right)-\Delta u(x, y) \\
= & -g\left(\left(x_{\lambda}, y\right), u\left(x_{\lambda}, y\right), v\left(x_{\lambda}, y\right)\right)+g((x, y), u(x, y), v(x, y)) \\
= & -g\left(\left(x_{\lambda}, y\right), u\left(x_{\lambda}, y\right), v\left(x_{\lambda}, y\right)\right)+g\left(\left(x_{\lambda}, y\right), u(x, y), v\left(x_{\lambda}, y\right)\right) \\
& \quad-g\left(\left(x_{\lambda}, y\right), u(x, y), v\left(x_{\lambda}, y\right)\right)+g\left(\left(x_{\lambda}, y\right), u(x, y), v(x, y)\right) \\
& \quad-g\left(\left(x_{\lambda}, y\right), u(x, y), v(x, y)\right)+g((x, y), u(x, y), v(x, y)) \\
=: & J_{1}+J_{2}+J_{3}
\end{aligned}
$$


It follows from $\left(H_{4}\right)$ that $J_{3}<0$. The other two expressions are evaluated using the Mean Value Theorem, namely

$$
\begin{aligned}
& J_{1}=-c_{\lambda}(x)\left[u\left(x_{\lambda}, y\right)-u(x, y)\right] \\
& J_{2}=-d_{\lambda}(x)\left[v\left(x_{\lambda}, y\right)-v(x, y)\right],
\end{aligned}
$$

where

$$
\begin{aligned}
& c_{\lambda}(x)=\frac{\partial g}{\partial t}\left(\left(x_{\lambda}, y\right), \zeta, v\left(x_{\lambda}, y\right)\right) \\
& d_{\lambda}(x)=\frac{\partial g}{\partial s}\left(\left(x_{\lambda}, y\right), u(x, y), \xi\right),
\end{aligned}
$$

where $\zeta$ is some value between $u\left(x_{\lambda}, y\right)$ and $u(x, y)$. Similarly for $\xi$.

Observe that $c_{\lambda}(x)$ and $d_{\lambda}(x)$ are non-negative in view of Hypothesis $\left(H_{3}\right)$. With a similar computation for $z_{\lambda}$, we come to the system:

$$
\begin{aligned}
& -\Delta w_{\lambda} \geq c_{\lambda} w_{\lambda}+d_{\lambda} z_{\lambda} \\
& -\Delta z_{\lambda} \geq c_{\lambda}^{\prime} w_{\lambda}+d_{\lambda}^{\prime} z_{\lambda}
\end{aligned}
$$

in a neighborhood of the boundary, where the coefficients are all nonnegative in that neighborhood. By the Maximum Principle for Cooperative Systems (see [16], [14]) $w_{\lambda}, z_{\lambda}$ are positive, and so there exists $\epsilon>0$ such that $u$ and $v$ are increasing in $\Omega_{\epsilon}$. At this point, the proof follows the same steps as in the proof of Theorem 1.1 in [10].

\section{Proof of Theorem 1.2:}

From Lemma 3.2 and an argument similar to the one in the proof of Theorem 1.1 in [10] we conclude that there exists $\varepsilon>0$ and $C>0$ such that

$$
\|u\|_{L^{\infty}}\left(\Omega_{\varepsilon}\right) \leq C,\|v\|_{L^{\infty}}\left(\Omega_{\varepsilon}\right) \leq C
$$

for all solutions $(u, v)$ of system (1.1).

Finally, let $a:=\inf \left\{\varphi_{1}^{b}(x) ; x \in \bar{\Omega} \backslash \Omega_{\varepsilon}\right\}$, and estimate

$$
\begin{aligned}
\int_{\Omega} f(x, u, v) \mathrm{d} x & =\int_{\Omega_{\varepsilon}} f(x, u, v) \mathrm{d} x+\int_{\Omega \backslash \Omega_{\varepsilon}} f(x, u, v) \mathrm{d} x \\
& \leq C+\frac{1}{a} \int_{\Omega \backslash \Omega_{\varepsilon}} f(x, u, v) \varphi_{1}^{b}(x) \mathrm{d} x \\
& \leq C+\frac{1}{a} \int_{\Omega} f(x, u, v) \varphi_{1}^{b}(x) \mathrm{d} x \leq C,
\end{aligned}
$$

by Lemma 3.1, and similarly for $\int_{\Omega} g(x, u, v) \mathrm{d} x$. This completes the proof of Theorem 1.2. 


\section{Proof of Theorem 1.3}

In this section we rely on two inequalities, some kind of Young inequalities. The first one was introduced in [11] to treat simpler elliptic systems in dimension two. The second one generalizes the first one to the case when there are three terms.

Proposition 4.1 For any $a, s, t \geq 1$ we have

$$
s t \leq e^{t^{a}}-1+s(\log s)^{1 / a} .
$$

Proposition 4.2 For any $a, b, r, s, t \geq 1$ we have

$$
r s t \leq e^{t^{a}}+e^{r^{b}}+s(\log s)^{\frac{1}{a}+\frac{1}{b}} .
$$

Proof. We are going to prove that for any $a, b, s \geq 1$ fixed we have

$$
\sup _{r, t>1}\left\{r s t-e^{t^{a}}-e^{r^{b}}\right\} \leq s(\log s)^{1 / a+1 / b} .
$$

Let $h(r, t):=r s t-e^{t^{a}}-e^{r^{b}}$. From

$$
\begin{aligned}
& \frac{\partial}{\partial r} h(r, t)=t_{s} s-b r_{s}^{b-1} e^{r_{s}^{b}}=0 \\
& \frac{\partial}{\partial t} h(r, t)=r_{s} s-a t_{s}^{a-1} e^{t_{s}^{a}}=0
\end{aligned}
$$

we obtain

$$
\log t_{s}+\log s=\log \left(b r_{s}^{b-1} e^{r_{s}^{b}}\right)=r_{s}^{b}+\log \left(b r_{s}^{b-1}\right) \geq r_{s}^{b},
$$

and

$$
\log r_{s}+\log s=\log \left(a t_{s}^{a-1} e^{t_{s}^{a}}\right)=t_{s}^{a}+\log \left(a t_{s}^{a-1}\right) \geq t_{s}^{a}
$$

using the fact that $a t_{s}^{a-1} \geq 1$ and $b t_{s}^{b-1} \geq 1$. Thus

$$
\begin{aligned}
& r_{s} \leq\left(\log t_{s}+\log s\right)^{1 / b} \\
& t_{s} \leq\left(\log r_{s}+\log s\right)^{1 / a}
\end{aligned}
$$

Therefore,

$$
\begin{aligned}
h(r, t) & \leq s r_{s} t_{s} \\
& \leq s\left(\log t_{s}+\log s\right)^{1 / b}\left(\log r_{s}+\log s\right)^{1 / a} \\
& \leq s(\log s)^{1 / a+1 / b}
\end{aligned}
$$


and the proof is complete.

\section{Proof of Theorem 1.3}

First we recall that Theorem 1.1 gives that each $(u, v)$ solution of (1.1) belongs to $\left(L^{\infty}(\bar{\Omega})\right)^{2}$, and then it follows that it belongs to $\left(W^{1,2}(\Omega)\right)^{2}$. Let $\eta, \eta^{\prime}$ be positive parameters to be determined later. Using the first equation of system (1.1) we obtain

$$
\begin{aligned}
\int_{\Omega}|\nabla u|^{2} \mathrm{~d} x & =\int_{\Omega} u g(x, u, v) \mathrm{d} x \\
& \leq c+\int_{\Omega_{1}} \frac{g(x, u, v)}{u^{\eta}+v^{\eta^{\prime}}}\left(u^{\eta}+v^{\eta^{\prime}}\right) u \\
& =: c+I_{1}+I_{2},
\end{aligned}
$$

where

$$
I_{1}:=\int_{\Omega_{1}} \frac{g(x, u, v)}{u^{\eta}+v^{\eta^{\prime}}} u^{\eta+1} \quad \text { and } \quad I_{2}:=\int_{\Omega_{1}} \frac{g(x, u, v)}{u^{\eta}+v^{\eta^{\prime}}} v^{\eta^{\prime}} u
$$

with

$$
\Omega_{1}:=\{x \in \Omega: u(x) \geq 1 \quad \text { or } \quad v(x) \geq 1\} .
$$

First we estimate $I_{1}$. We have

$$
I_{1} \leq\|u\|^{\eta+1} \int_{\Omega_{1}} \frac{g(x, u, v)}{u^{\eta}+v^{\eta^{\prime}}}\left(\frac{u}{\|u\|}\right)^{\eta+1},
$$

and using Proposition 4.1 with $a=\gamma$, we obtain

$$
I_{1} \leq\|u\|^{\eta+1}\left\{\int_{\Omega_{1}} e^{\left(\frac{u}{\|u\|}\right)^{(\eta+1) \gamma}}+\int_{\Omega_{1}} \frac{g(x, u, v)}{u^{\eta}+v^{\eta^{\prime}}}\left(\log \frac{g(x, u, v)}{u^{\eta}+v^{\eta^{\prime}}}\right)^{1 / \gamma}\right\} .
$$

The first term above is bounded by Trudinger-Moser inequality, if we take

$$
(\eta+1) \gamma \leq 2 \text {. }
$$

To estimate the second term we notice that by assumption $\left(H_{6}\right)$

$$
\begin{aligned}
& \int_{\Omega_{1}} \frac{g(x, u, v)}{u^{\eta}+v^{\eta^{\prime}}}\left(\log \frac{g(x, u, v)}{u^{\eta}+v^{\eta^{\prime}}}\right)^{1 / \gamma} \leq \int_{\Omega_{1}} \frac{g(x, u, v)}{u^{\eta}+v^{\eta^{\prime}}}\left(\log \left(e^{u^{\alpha}+v^{\beta}}\right)\right)^{1 / \gamma} \\
& \leq C \int_{\Omega_{1}} \frac{g(x, u, v)}{u^{\eta}+v^{\eta^{\prime}}}\left(u^{\alpha / \gamma}+v^{\beta / \gamma}\right) \leq C \int_{\Omega_{1}} g(x, u, v) \leq C
\end{aligned}
$$


if we take

$$
\eta \geq \alpha / \gamma \text { and } \eta^{\prime} \geq \beta / \gamma,
$$

and where we have used the elementary inequality

$$
\left(u^{\alpha}+v^{\beta}\right)^{1 / \gamma} \leq C\left(u^{\alpha / \gamma}+v^{\beta / \gamma}\right) .
$$

Thus we have

$$
I_{1} \leq C\left(\|u\|^{\eta+1}+1\right) .
$$

Next we estimate $I_{2}$. By Proposition 4.2 we get

$$
\begin{aligned}
I_{2} & \leq C\|u\|\|v\|^{\eta^{\prime}} \int_{\Omega_{1}} \frac{g(x, u, v)}{u^{\eta}+v^{\eta^{\prime}}}\left(\frac{v}{\|v\|}\right)^{\eta^{\prime}}\left(\frac{u}{\|u\|}\right) \\
& \leq C\|u\|\|v\|^{\eta^{\prime}}\left\{\int_{\Omega_{1}} e^{\left(\frac{v}{\|v\|}\right)^{\eta^{\prime} a}}+\int_{\Omega_{1}} e^{\left(\frac{u}{\|u\|}\right)^{b}}+J_{1}\right\},
\end{aligned}
$$

where

$$
J_{1}=\int_{\Omega_{1}} \frac{g(x, u, v)}{u^{\eta}+v^{\eta^{\prime}}} \log \left(\frac{g(x, u, v)}{u^{\eta}+v^{\eta^{\prime}}}\right)^{\left(a^{-1}+b^{-1}\right)} .
$$

Using Trudinger-Moser inequality and taking

$$
\eta^{\prime} a \leq 2 \text { and } \quad b \leq 2
$$

we obtain

$$
I_{2} \leq C\|u\|\|v\|^{\eta^{\prime}}\left\{C+J_{1}\right\}
$$

Finally, the integral $J_{1}$ is estimated as above:

$$
\begin{aligned}
J_{1} & \leq \int_{\Omega_{1}} \frac{g(x, u, v)}{u^{\eta}+v^{\eta^{\prime}}}\left(u^{\alpha\left(a^{-1}+b^{-1}\right)}+v^{\beta\left(a^{-1}+b^{-1}\right)}\right) \\
& \leq C \int_{\Omega_{1}} g(x, u, v) \mathrm{d} x \leq C,
\end{aligned}
$$

by choosing

$$
\alpha\left(\frac{1}{a}+\frac{1}{b}\right) \leq \eta \text { and } \beta\left(\frac{1}{a}+\frac{1}{b}\right) \leq \eta^{\prime} .
$$

Thus, we have obtained

$$
I_{2} \leq C\|v\|^{\eta^{\prime}}\|u\| .
$$

Hence, altogether with the above choices we have

$$
\|u\|^{2}=\int_{\Omega}|\nabla u|^{2} \leq C\left(\|u\|^{\eta+1}+\|v\|^{\eta^{\prime}}\|u\|+1\right),
$$


which implies

$$
\|u\| \leq C\left(\|u\|^{\eta}+\|v\|^{\eta^{\prime}}+1\right) .
$$

We now analyze the inequalities (4.1) - (4.2), viewing to obtain the possible values of the parameters $\eta$ and $\eta^{\prime}$. First, we eliminate $\gamma, a$ and $b$ : by using (4.1) - (4.2) we obtain

$$
\eta \geq \frac{\alpha}{2-\alpha}
$$

and

$$
\eta^{\prime} \geq \frac{\beta(\eta+1)}{2}
$$

By using (4.3) and (4.4) we have

$$
\begin{aligned}
& \frac{1}{a}+\frac{1}{b} \geq \frac{\eta^{\prime}+1}{2} . \\
& \eta \geq \frac{\alpha\left(\eta^{\prime}+1\right)}{2}
\end{aligned}
$$

and

$$
\eta^{\prime} \geq \frac{\beta}{2-\beta}
$$

We now choose equality in $(I I I)$ and $(I V)$ to obtain $\eta^{\prime}$ :

$$
\eta^{\prime}=\frac{\beta}{2-\beta}
$$

and

$$
\eta=\frac{\alpha}{2-\beta} .
$$

Checking the compatibility of this choice with inequality $(I)$ yields:

$$
\frac{\alpha}{2-\beta}=\eta \geq \frac{\alpha}{2-\alpha},
$$

which agrees with the assumption $\left(H_{6}, a\right)$, that is, $\alpha \leq \beta$, which is also compatible with $(I I)$.

We now return to inequality (4.5). To eliminate the term $\|u\|^{\eta}$ on the right, we need to require

$$
\eta<1 \Longleftrightarrow \alpha+\beta<2, \text { that is, hypothesis }\left(H_{6}, b\right) ;
$$


thus, inequality (4.5) reduces to

$$
\|u\| \leq C\left(\|v\|^{\eta^{\prime}}+1\right) .
$$

Note that $2>\alpha+\beta \geq 2 \alpha$ also implies that $1>\alpha$.

Next, we proceed similarly with the second equation in system (1.1)

$$
\begin{aligned}
\int_{\Omega}|\nabla v|^{2} \mathrm{~d} x & =\int_{\Omega} f(x, u, v) v \\
& \leq c+\int_{\Omega_{1}} \frac{f(x, u, v)}{u^{\bar{\eta}^{\prime}}+v^{\bar{\eta}}}\left(u^{\bar{\eta}^{\prime}}+v^{\bar{\eta}}\right) v \\
& =c+J_{1}+J_{2}
\end{aligned}
$$

where

$$
J_{1}=\int_{\Omega_{1}} \frac{f(x, u, v)}{u^{\bar{\eta}^{\prime}}+v^{\bar{\eta}}} v^{\bar{\eta}+1} .
$$

We estimate as before, using Proposition 4.1, the Trudinger-Moser inequality and Theorem 1.2

$$
\begin{aligned}
J_{1} & \leq C\|v\|^{\bar{\eta}+1} \int_{\Omega_{1}} \frac{f(x, u, v)}{u^{\bar{\eta}^{\prime}}+v^{\bar{\eta}}}\left(\frac{v}{\|v\|}\right)^{\bar{\eta}+1} \\
& \leq C\|v\|^{\bar{\eta}+1}\left\{\int_{\Omega_{1}} e^{\left(\frac{v}{\|v\|}\right)^{(\bar{\eta}+1) \gamma^{\prime}}}+\int_{\Omega_{1}} \frac{f(x, u, v)}{u^{\bar{\eta}^{\prime}}+v^{\bar{\eta}}}\left(\log \frac{f(x, u, v)}{u^{\bar{\eta}^{\prime}}+v^{\bar{\eta}}}\right)^{1 / \gamma^{\prime}}\right\} \\
& \leq C\|v\|^{\bar{\eta}+1},
\end{aligned}
$$

if we choose

$$
(\bar{\eta}+1) \gamma^{\prime} \leq 2, \quad \bar{\eta}^{\prime} \geq \frac{\beta^{\prime}}{\gamma^{\prime}}, \quad \bar{\eta} \geq \frac{\alpha^{\prime}}{\gamma^{\prime}} .
$$

The integral $J_{2}$ is estimated using Proposition 4.2, Trudinger-Moser inequality and Theorem 1.2

$$
\begin{aligned}
& J_{2} \leq c \int_{\Omega_{1}} \frac{f(x, u, v)}{u^{\bar{\eta}^{\prime}}+v^{\bar{\eta}}} u^{\bar{\eta}^{\prime}} v=c\|u\|^{\bar{\eta}^{\prime}}\|v\| \int_{\Omega_{1}} \frac{f(x, u, v)}{u^{\bar{\eta}^{\prime}}+v^{\bar{\eta}}}\left(\frac{u}{\|u\|}\right)^{\bar{\eta}^{\prime}}\left(\frac{v}{\|v\|}\right) \\
& \leq c\left\{\int_{\Omega_{1}} e^{\left(\frac{u}{\|u\|} \bar{\eta}^{\bar{\eta}^{\prime} a}\right.} \mathrm{d} x+\int_{\Omega_{1}} e^{\left(\frac{v}{\|v\|}\right)^{b^{\prime}}} \mathrm{d} x+\int_{\Omega_{1}} \frac{f(x, u, v)}{u^{\bar{\eta}^{\prime}}+v^{\bar{\eta}}} \log \left(\frac{f(x, u, v)}{u^{\bar{\eta}^{\prime}}+v^{\bar{\eta}}}\right)^{\frac{1}{a^{\prime}}+\frac{1}{b^{\prime}}}\right\} \\
& \leq C\|u\|^{\bar{\eta}^{\prime}}\|v\|,
\end{aligned}
$$

if we choose

$$
\bar{\eta}^{\prime} a^{\prime} \leq 2 \quad, \quad b^{\prime} \leq 2 \quad, \quad \bar{\eta}^{\prime} \geq \beta^{\prime}\left(\frac{1}{a^{\prime}}+\frac{1}{b^{\prime}}\right) \quad, \quad \bar{\eta} \geq \alpha^{\prime}\left(\frac{1}{a^{\prime}}+\frac{1}{b^{\prime}}\right)
$$


Joining the estimates for $J_{1}$ and $J_{2}$ we thus obtain

$$
\|v\|^{2} \leq c\|v\|^{\bar{\eta}+1}+c\|u\|^{\bar{\eta}^{\prime}}\|v\|+c
$$

that is,

$$
\|v\| \leq c\|v\|^{\bar{\eta}}+c\|u\|^{\bar{\eta}^{\prime}}+c .
$$

¿From (4.9) and (4.10) we now get, by choosing equality in the appropriate relations, the analogues of (4.6) and (4.7)

$$
\bar{\eta}^{\prime}=\frac{\beta^{\prime}}{2-\beta^{\prime}}, \bar{\eta}=\frac{\alpha^{\prime}}{2-\beta^{\prime}}
$$

By condition $\left(H_{6}, b\right)$ we conclude that $\bar{\eta}<1$, and then we obtain from (4.11) that

$$
\|v\| \leq C\left(\|u\|^{\bar{\eta}^{\prime}}+1\right)
$$

Finally, joining inequalities (4.8) and (4.12) we get

$$
\|u\| \leq C\left(\|v\|^{\eta^{\prime}}+1\right) \leq C\left(\|u\|^{\eta^{\prime} \bar{\eta}^{\prime}}+1\right)
$$

The condition $\left(H_{6}, c\right)$ yields $\eta^{\prime} \bar{\eta}^{\prime}<1$, since

$$
\begin{aligned}
\eta^{\prime} \bar{\eta}^{\prime}<1 & \Longleftrightarrow \frac{\beta}{2-\beta} \frac{\beta^{\prime}}{2-\beta^{\prime}}<1 \\
& \Longleftrightarrow \beta \beta^{\prime}<4-2 \beta-2 \beta^{\prime}+\beta \beta^{\prime} \\
& \Longleftrightarrow \beta+\beta^{\prime}<2,
\end{aligned}
$$

and hence the solutions $u$ are uniformly bounded in $H_{0}^{1}(\Omega)$. Joining inequalities (4.8) and (4.12) in the other order we obtain that also the solutions $v$ are uniformly bounded. Finally, since $e^{t} \leq c_{\varepsilon} e^{\varepsilon t^{2}}$ for any $\varepsilon>0$, we conclude by the Trudinger-Moser inequality that the righthand sides in system (1.1) are uniformly bounded in $L^{p}$, for any $p>1$, and hence we conclude that all solutions $(u, v)$ are uniformly bounded in $L^{\infty}$.

\section{Proof of Theorem 1.4}

Assume that $\left(u_{n}, v_{n}\right)$ is a sequence of solutions of system (1.1). Theorem 1.2 says that the sequences $\left(f\left(x, u_{n}, v_{n}\right)\right)$ and $\left(g\left(x, u_{n}, v_{n}\right)\right)$ are bounded in $L^{1}(\Omega)$. So it follows, passing to subsequences if necessary, (see [4]) that there is a nonnegative Radon measure $\mu$ such that

$$
f\left(x, u_{n}, v_{n}\right)+g\left(x, u_{n}, v_{n}\right) \rightarrow \mu .
$$


We also observe that, as a consequence of Theorem 1.2 and assumption $\left(H_{2}\right)$ (or $\left.\left(H_{2}^{\prime}\right)\right)$, the solutions $\left(\left(u_{n}, v_{n}\right)\right)$ of $(1.1)$ are bounded in $L^{1}(\Omega)$ :

$$
\left\|u_{n}\right\|_{L^{1}},\left\|v_{n}\right\|_{L^{1}} \leq C, \text { for all } n \text {. }
$$

Definition 5.1 We say that $x_{0} \in \Omega$ is a regular point of the measure $\mu$ if there is a function $\psi \in C_{c}(\Omega), 0 \leq \psi \leq 1$, with $\psi \equiv 1$ in some neighborhood of $x_{0}$ and such that

$$
\int_{\Omega} \psi d \mu<4 \pi
$$

We denote by $\Sigma_{\mu}$ the set of non-regular points in $\Omega$ for the measure $\mu$.

Remark 5.1 For a bounded non-negative measure $\mu, \Sigma_{\mu}$ is a finite set. Indeed, if $x_{0} \in \Sigma_{\mu}$, we have that

$$
\int_{\Omega} \psi d \mu \geq 4 \pi
$$

for any function $\psi \in C_{c}(\Omega), 0 \leq \psi \leq 1$, with $\psi \equiv 1$ in some neighborhood of $x_{0}$. Thus, $\mu\left(\left\{x_{0}\right\}\right) \geq 4 \pi$. Finally, since $\int_{\Omega} d \mu \leq C$, it follows that $\Sigma_{\mu}$ is a finite set.

Let $S_{u}$ be the blow-up set for the sequence $\left(u_{n}\right)$, that is

$$
S_{u}:=\left\{x \in \Omega: \exists\left(x_{n}\right) \subset \Omega \text { such that } x_{n} \rightarrow x \text { and } u_{n}\left(x_{n}\right) \rightarrow+\infty\right\} .
$$

The assertions of Theorem 1.4 will be proved if we show that $S_{u}=S_{v}=$ $\emptyset$. This will be achieved in the next lemmas.

Lemma 5.1 Assume that $x_{0}$ is a regular point for the measure $\mu$. Then there exist constants $\rho>0$ and $C$, independent of $n$, such that

$$
\left\|u_{n}\right\|_{L^{\infty}\left(B_{\rho}\left(x_{0}\right)\right)} \leq C,\left\|v_{n}\right\|_{L^{\infty}\left(B_{\rho}\left(x_{0}\right)\right)} \leq C .
$$

Proof. Using the fact that $x_{0}$ is a regular point of the measure $\mu$ we have a function $\psi \in C_{c}(\Omega), 0 \leq \psi \leq 1$, with $\psi \equiv 1$ in some neighborhood $V_{x_{0}}$ of $x_{0}$, such that $\int \psi d \mu<4 \pi$. Thus, $\int_{V_{x_{0}}} d \mu<4 \pi$, which implies that there exist $R>0, \delta>0$ and $n_{0}$ such that for all $n \geq n_{0}$,

$$
\int_{B_{R}\left(x_{0}\right)}\left[f\left(x, u_{n}, v_{n}\right)+g\left(x, u_{n}, v_{n}\right)\right] \mathrm{d} x \leq 4 \pi-\delta .
$$


Let us write $u_{n}:=u_{1, n}+u_{2, n}$ and $v_{n}:=v_{1, n}+v_{2, n}$, where

$$
-\Delta u_{1, n}=g\left(x, u_{n}, v_{n}\right), \text { in } B_{R}\left(x_{0}\right) \text { and } u_{1, n}=0 \text { for }\left|x-x_{0}\right|=R
$$

and

$$
-\Delta v_{1, n}=f\left(x, u_{n}, v_{n}\right) \text {, in } B_{R}\left(x_{0}\right) \text { and } v_{1, n}=0 \text { for }\left|x-x_{0}\right|=R
$$

Notice that $-\Delta u_{2, n}=0$ and $-\Delta v_{2, n}=0$ in $B_{R}\left(x_{0}\right)$.

Using Proposition 2.1 and (5.3), we obtain

$$
c \geq \int_{B_{R}} e^{\left(4 \pi-\frac{\delta}{2}\right) \frac{u_{1, n}+v_{1, n}}{\int(f+g)}} \geq \int_{B_{R}} e^{\left(4 \pi-\frac{\delta}{2}\right) \frac{u_{1, n}+v_{1, n}}{4 \pi-\delta}}=\int_{B_{R}} e^{p\left(u_{1, n}+v_{1, n}\right)},
$$

where $p>1$ is a constant depending only on $\delta$. Using the fact that $t \leq e^{t}$ we get

$$
\left\|u_{1, n}\right\|_{L^{1}\left(B_{R}\left(x_{0}\right)\right)} \leq C \quad \text { and } \quad\left\|v_{1, n}\right\|_{L^{1}\left(B_{R}\left(x_{0}\right)\right)} \leq C .
$$

Furthermore, as in the regularity Theorem 1.1 we get

$$
\left\|u_{1, n}\right\|_{L^{\infty}\left(B_{R}\left(x_{0}\right)\right)} \leq C \quad \text { and } \quad\left\|v_{1, n}\right\|_{L^{\infty}\left(B_{R}\left(x_{0}\right)\right)} \leq C .
$$

Since the functions $u_{2, n}, v_{2, n}$ are harmonic in $B_{R}\left(x_{0}\right)$, it follows from the Mean Value Theorem for harmonic functions that

$$
\left\|u_{2, n}\right\|_{L^{\infty}\left(B_{R / 2}\right)} \leq C\left\|u_{2, n}\right\|_{L^{1}\left(B_{R}\right)} \text { and }\left\|v_{2, n}\right\|_{L^{\infty}\left(B_{R / 2}\right)} \leq C\left\|v_{2, n}\right\|_{L^{1}\left(B_{R}\right)} .
$$

On the other hand, using (5.2) and (5.5) we obtain

$$
\left\|u_{2, n}\right\|_{L^{1}\left(B_{R}\right)} \leq\left\|u_{n}\right\|_{L^{1}\left(B_{R}\right)}+\left\|u_{1, n}\right\|_{L^{1}\left(B_{R}\right)} \leq C
$$

and

$$
\left\|v_{2, n}\right\|_{L^{1}\left(B_{R}\right)} \leq\left\|v_{n}\right\|_{L^{1}\left(B_{R}\right)}+\left\|v_{1, n}\right\|_{L^{1}\left(B_{R}\right)} \leq C .
$$

Thus

$$
\left\|u_{2, n}\right\|_{L^{\infty}\left(B_{R / 2}\right)} \leq C \quad \text { and } \quad\left\|v_{2, n}\right\|_{L^{\infty}\left(B_{R / 2}\right)} \leq C .
$$

From (5.6) and (5.7) we obtain

$$
\left\|u_{n}\right\|_{L^{\infty}\left(B_{R / 2}\right)} \leq C \quad \text { and } \quad\left\|v_{n}\right\|_{L^{\infty}\left(B_{R / 2}\right)} \leq C .
$$

and the proof is complete.

Lemma $5.2 S_{u} \cup S_{v} \subset \Sigma_{\mu}$. 
Proof. Suppose that $x_{0} \notin \Sigma_{\mu}$, i.e. $x_{0}$ is a regular point for $\mu$. Then it follows by Lemma 5.1 that $\left\|u_{n}\right\|_{L^{\infty}\left(B_{\rho}\left(x_{0}\right)\right)} \leq C$ and $\left\|v_{n}\right\|_{L^{\infty}\left(B_{\rho}\left(x_{0}\right)\right)} \leq C$ for some $\rho>0$, and hence $x_{0} \notin S_{u} \cup S_{v}$ by the definition of the sets $S_{u}$ and $S_{v}$.

Lemma $5.3 \Sigma_{\mu} \subset S_{u} \cup S_{v}$.

Proof. Let $x_{0} \in \Sigma_{\mu}$. We claim that for each $R>0$ we have

$$
\lim _{n \rightarrow+\infty}\left\|u_{n}\right\|_{L^{\infty}\left(B_{R}\left(x_{0}\right)\right)}=+\infty \quad \text { or } \quad \lim _{n \rightarrow+\infty}\left\|v_{n}\right\|_{L^{\infty}\left(B_{R}\left(x_{0}\right)\right)}=+\infty
$$

Suppose by contradiction that there exists $R_{0}>0$ and a subsequence, which we denote also by $\left(u_{n}\right),\left(v_{n}\right)$, such that

$$
\left\|u_{n}\right\|_{L^{\infty}\left(B_{R_{0}}\left(x_{0}\right)\right)} \leq C \quad \text { and } \quad\left\|v_{n}\right\|_{L^{\infty}\left(B_{R_{0}}\left(x_{0}\right)\right)} \leq C .
$$

So,

$$
\left\|e^{u_{n}+v_{n}}\right\|_{L^{\infty}\left(B_{R_{0}}\left(x_{0}\right)\right)} \leq C .
$$

Now using the hypothesis

$$
f(x, u, v) \leq d e^{t+s} \quad, \quad g\left(x, u, v \leq d e^{u+v}\right.
$$

it follows that

$$
\left\|f\left(x, u_{n}, v_{n}\right)\right\|_{L^{\infty}\left(B_{R_{0}}\left(x_{0}\right)\right)} \leq C \quad \text { and } \quad\left\|g\left(x, u_{n}, v_{n}\right)\right\|_{L^{\infty}\left(B_{R_{0}}\left(x_{0}\right)\right)} \leq C
$$

which implies that for $R<R_{0}$ we have

$$
\int_{B_{R}\left(x_{0}\right)}\left[f\left(x, u_{n}, v_{n}\right)+g\left(x, u_{n}, v_{n}\right)\right] \leq C R^{2}
$$

Thus, there exists $R_{1}>0$, such that

$$
\int_{B_{R_{1}}\left(x_{0}\right)}\left[f\left(x, u_{n}, v_{n}\right)+g\left(x, u_{n}, v_{n}\right)\right]<4 \pi .
$$

This implies that $x_{0}$ is a regular point of $\mu$, which is a contradiction.

As a consequence of Lemmas 5.2 and 5.3 we conclude that

$$
\Sigma_{\mu}=S_{u} \cup S_{v} .
$$

Finally, we prove that this set is indeed empty, and this completes the proof of the Theorem 1.4. 
Lemma $5.4 S_{u} \cup S_{v}=\emptyset$.

Proof. Suppose, by contradiction, that there exists $x_{0} \in S_{u} \cup S_{v}$. Since $x_{0}$ is isolated, we can take $R>0$ such that $\overline{B_{R}\left(x_{0}\right)} \cap\left(\left(S_{u} \cup S_{v}\right) \backslash\left\{x_{0}\right\}\right)=\emptyset$.

Next, we consider the Dirichlet problem in $B_{R}\left(x_{0}\right)$,

$-\Delta z_{n}=f\left(x, u_{n}, v_{n}\right)+g\left(x, u_{n}, v_{n}\right)$, in $B_{R}\left(x_{0}\right)$, and $z_{n}=0$ for $\left|x-x_{0}\right|=R$.

We know that the function $u_{n}+v_{n}$ satisfies

$-\Delta\left(u_{n}+v_{n}\right)=f\left(x, u_{n}, v_{n}\right)+g\left(x, u_{n}, v_{n}\right)$, in $B_{R}\left(x_{0}\right)$, and $u_{n}+v_{n} \geq 0$ for $\left|x-x_{0}\right|=R$.

Thus, by the Maximum Principle we have

$$
0 \leq z_{n} \leq u_{n}+v_{n} \text { in } \overline{B_{R}\left(x_{0}\right)}
$$

Taking the limit we have $z_{n} \rightarrow z$, where $z$ is a solution of the problem

$$
-\Delta z=\mu \text {, in } B_{R}\left(x_{0}\right) \text { and } z=0 \text { when }\left|x-x_{0}\right|=R .
$$

On the other hand the problem

$$
-\Delta w=4 \pi \delta_{0} \text { in } B_{R}\left(x_{0}\right) \text { and } w=0 \text { when }\left|x-x_{0}\right|=R
$$

has the solution

$$
w(x)=2 \log \frac{R}{\left|x-x_{0}\right|} .
$$

Since $x_{0}$ is not a regular point of $\mu$ it follows that $\mu \geq 4 \pi \delta_{0}$. So

$$
z(x) \geq 2 \log \left|x-x_{0}\right|^{-1}+o(1), x \rightarrow x_{0}
$$

Now, by hypothesis $\left(H_{7}\right)$

$$
f(x, t, s)+g(x, t, s) \geq g(x, t, s) \geq c e^{t+s},
$$

we have

$$
\begin{gathered}
\lim _{n \rightarrow \infty} \int_{B_{R}\left(x_{0}\right)}\left[f\left(x, u_{n}, v_{n}\right)+g\left(x, u_{n}, v_{n}\right)\right] \mathrm{d} x \geq \\
\geq \lim _{n \rightarrow \infty} c \int_{B_{R}\left(x_{0}\right)} e^{z_{n}} \\
\geq c \int_{B_{R}\left(x_{0}\right)} e^{w}=\infty
\end{gathered}
$$

which is impossible. 


\section{Proof of Theorem 1.5}

We rely on the following well-known Theorem by Krasnoselsk'ii (we state in the form given in [8], Theorem 3.1).

\section{Theorem 6.1}

Let $C \subset E$ denote a cone in a Banach space, and let $T: C \rightarrow C$ be a compact mapping, with $T(0)=0$. Furthermore, assume that there are real numbers $0<r<R$ such that

(1) $x \neq T x$, for $0 \leq t \leq 1,\|x\|=r, x \in C$

(2) there exists $H: C \times[0, \infty) \rightarrow C$ such that

(a) $H(x, 0)=T x, x \in C$

(b) $H(x, t) \neq x,\|x\|=R, t \geq 0$

(c) $H(x, t)=x$ has no solution $x \in \bar{B}_{R}, t \geq t_{0}$

Then (1) implies $i_{C}\left(T, B_{r}\right)=1$, and (2) implies $i_{C}\left(T, B_{R}\right)=0$.

Here $i_{C}(T, \mathcal{O})$ is the fixed point index of $T$ in $C$ with respect to the (relatively) open set $\mathcal{O}$.

Note that it then follows by the excision property that $T$ has a fixed point in $B_{R} \backslash \bar{B}_{r}$.

Proof of Theorem 1.5:

Let $X=C^{0}(\bar{\Omega}) \times C^{0}(\bar{\Omega})$ with norm $\|(u, v)\|=\|u\|_{\infty}+\|v\|_{\infty}$, and set

$$
C=\{(u, v) \in X ; u \geq 0, v \geq 0\}
$$

Furthermore, define the compact mapping $T: C \rightarrow C$ given by

$$
T\left(\begin{array}{l}
u \\
v
\end{array}\right)=\left(\begin{array}{c}
(-\Delta)^{-1} g(x, u, v) \\
(-\Delta)^{-1} f(x, u, v)
\end{array}\right)
$$

We now check that $T$ satisfies hypotheses $(i)$ and $(i i)$ of Theorem 1.5:

Hypthesis (1): We have to show that there is no solution $(u, v)$ of

$$
\left\{\begin{array}{l}
-\Delta u=t g(x, u, v) \\
-\Delta v=t f(x, u, v)
\end{array} \quad, \text { for } \quad 0 \leq t \leq 1,\|(u, v)\|=r, r<\varepsilon,\right.
$$


where $\varepsilon$ is given in $\left(H_{8}\right)$.

Indeed, suppose by contradiction that there is a solution $(u, v) \in C$ with $\|(u, v)\|=r$. Then we can estimate by $\left(H_{8}\right)$ the righthand side of (5.10)

$$
\begin{aligned}
\|t g(x, u, v)\|_{\infty} & \leq c_{1}\|(u, v)\|=c_{1} r \\
\|t f(x, u, v)\|_{\infty} & \leq c_{2} r
\end{aligned}
$$

and obtain by (5.10) and (1.7)

$$
\|u\|_{\infty} \leq M c_{1} r,\|v\|_{\infty} \leq M c_{2} r .
$$

This implies that

$$
r=\|(u, v)\|=\|u\|_{\infty}+\|v\|_{\infty} \leq M\left(c_{1}+c_{2}\right) r,
$$

which is a contradiction, since $M\left(c_{1}+c_{2}\right)<1$.

Hypothesis (2):

Define the homotopy $H: C \times[0,1] \rightarrow C$

$$
H\left(\left(\begin{array}{c}
u \\
v
\end{array}\right), t\right)=\left(\begin{array}{c}
(-\Delta)^{-1} g_{t}((x, u, v) \\
(-\Delta)^{-1} f_{t}(x, u, v)
\end{array}\right),
$$

where

$$
\begin{aligned}
& g_{t}(x, u, v)=(1-t) g(x, u, v)+t \mu\left(v^{+}+1\right) \\
& f_{t}(x, u, v)=(1-t) f(x, u, v)+t \mu\left(u^{+}+1\right)
\end{aligned},
$$

with $\mu>\max \left\{\lambda_{1},\|a\|_{\infty},\|b\|_{\infty}\right\}$, where $a, b$ are as in assumption $\left(H_{2}\right)$, resp. assumption $\left(H_{2}^{\prime}\right)$.

Note that with this choice $g_{t}$ and $f_{t}$ satisfy assumption $\left(H_{2}\right)$ uniformly with respect to $t$; indeed, for $u, v \geq 0$

$$
\begin{aligned}
g_{t}(x, u, v) & \geq(1-t)\left(b(x) v-b_{1}(x)\right)+t \mu(v+1) \\
& =((1-t) b(x)+t \mu) v-(1-t) b_{1}(x)+t \mu \\
& \geq b(x) v-b_{1}(x)+t b_{1}(x)+t \mu \\
& \geq b(x) v-b_{1}(x)
\end{aligned}
$$

and similarly for $f_{t}(x, u, v)$.

Observe that the hypotheses on $f$ and $g$ transfer to $f_{t}$ and $g_{t}$ with the same parameters. So $(2, \mathrm{~b})$ holds for $R$ sufficiently large by the a priori bounds for solutions in Theorem 1.3 and Theorem 1.4. 
Finally, we show $(2, \mathrm{c})$, that is, that there exists no solution for $t=1$ : otherwise we would have

$$
\begin{aligned}
-\Delta u & =\mu\left(v^{+}+1\right) \\
-\Delta v & =\mu\left(u^{+}+1\right)
\end{aligned} \quad \Longrightarrow \quad \begin{aligned}
-\Delta u & =\mu v+\mu \\
-\Delta v & =\mu u+\mu
\end{aligned}
$$

Multiplying both equations by the first eigenfunction $\varphi_{1}$ and integration gives

$$
\begin{aligned}
& \lambda_{1} \int_{\Omega} u \varphi_{1}=\mu \int_{\Omega} v \varphi_{1}+\mu \int_{\Omega} \varphi_{1} \\
& \lambda_{1} \int_{\Omega} v \varphi_{1}=\mu \int_{\Omega} u \varphi_{1}+\mu \int_{\Omega} \varphi_{1}
\end{aligned}
$$

Inserting the second equation into the first we obtain

$$
\lambda_{1} \int_{\Omega} u \varphi_{1}=\mu\left\{\frac{\mu}{\lambda_{1}} \int_{\Omega} u \varphi_{1}+\frac{\mu}{\lambda_{1}} \int_{\Omega} \varphi_{1}\right\}+\mu \int_{\Omega} \varphi_{1}
$$

and hence

$$
\lambda_{1}^{2} \int_{\Omega} u \varphi_{1}=\mu^{2} \int_{\Omega} u \varphi_{1}+\mu^{2} \int_{\Omega} \varphi_{1}+\mu \lambda_{1} \int_{\Omega} \varphi_{1}
$$

this is a contradiction, since $\mu>\lambda_{1}$.

Hence, the map $T$ given in (5.9) has a nontrivial fixed point $(u, v)$ in $C$, that is, $(u, v)$ is a positive solution of system (1.1).

\section{References}

[1] R.A. Adams, J.F. Fournier, Sobolev spaces, second ed., Academic Press, 2003.

[2] H. Berestycki, L. Nirenberg, On the method of moving planes and the sliding method, Bol. Soc. Brasil. Mat. (N.S.) 22 (1991), 1-37.

[3] H. Brezis, R.E.L. Turner, On a class of superlinear elliptic problems, Comm. Partial Differential Equations 2 (1977), 601-614.

[4] H. Brezis, Analyse fonctionnelle, Théorie et applications, Masson, Paris, 1983. 
[5] H. Brezis, F. Merle, Uniform estimates and blow-up behavior for solutions of $-\Delta u=V(x) e^{u}$ in two dimensions, Comm. Partial Differential Equations 16 (1991), 1223-1253.

[6] Wen Xiong Chen, Congming Li, A priori estimates for solutions to nonlinear elliptic equations, Arch. Rational Mech. Anal. 122 (1993), $145-157$.

[7] D.G. de Figueiredo, Semilinear Elliptic Systems: Existence, Multiplicity, Symmetry of Solutions Handbook of Differential Equations, vol. 5: Stationary Partial Differential Equations, Editor M. Chipot, Elsevier, 2008

[8] D.G. de Figueiredo, Positive soutions of semilinear ellpitic equations, Lecture Notes in Mathematics No. 957, Springer-Verlag, 1982

[9] D.G. de Figueiredo, Monotonicity and symmetry of solutions of elliptic systems in general domains, NoDEA Nonlinear Differential Equations Appl. 1 (1994), 119-123.

[10] D.G. de Figueiredo, P.-L Lions, R. D. Nussbaum, A priori estimates and existence of positive solutions of semilinear elliptic equations, J. Math. Pures Appl. 61 (1982), 41-63.

[11] D.G. de Figueiredo, João Marcos do Ó and Bernhard Ruf, Critical and subcritical elliptic systems in dimension two, Indiana Univ. Math. J. 53 (2004), 1037-1054.

[12] D.G. de Figueiredo, João Marcos do Ó and Bernhard Ruf, An Orliczspace approach to superlinear elliptic systems, J. Funct. Anal. 224 (2005), no. 2, 471-496.

[13] D.G. de Figueiredo, João Marcos do Ó and Bernhard Ruf, Semilinear elliptic systems with exponential nonlinearities in two dimensions, Adv. Nonlinear Stud. 6 (2006), 199-213.

[14] D.G. de Figueiredo and Enzo Mitidieri, Maximum Principles for Linear Elliptic Systems, Rendiconti dell'Istituto di Matematica del'Universita di Trieste XXII, fasc. I e II, (1990), 36-66.

[15] J. Moser, A sharp form of an inequality by N. Trudinger, Ind. Univ. J. 20 (1971), 1077-1092. 
[16] M. Protter and H. Weinberger, Maximum principle in Differential Equations, Prentice-Hall, 1967.

[17] B. Ruf, Lorentz spaces and nonlinear elliptic systems. Contributions to nonlinear analysis, 471-489, Progr. Nonlinear Differential Equations Appl., 66, Birkhäuser, Basel, 2006.

[18] B. Ruf Superlinear Elliptic Equations and Systems, Handbook of Differential Equations, vol. 5: Stationary Partial Differential Equations, Editor M. Chipot, Elsevier, 2008

[19] P. Souplet, Optimal regularity conditions for elliptic problems via $L_{\delta}^{p}$ spaces, Duke Math. J. 127, 1, (2005)

[20] W. Troy, Symmetry properties in systems of semilinear elliptic equations, J. Differential Equations 42 (1981), 400-413. 